DOI: https://doi.org/10.46296/rc.v4i7.0025

\title{
La Corresponsabilidad Familiar en la Restitución de Derechos de los Adolescentes en Conflicto con la Ley en el Cantón Guayaquil
}

\section{Family Co-responsibility in the Restitution of the Rights of Adolescents in Conflict with the Law in the Canton Guayaquil}

\author{
Autora: Álvarez-Santana Carmita \\ Doctora en Desarrollo Local y Economía Social, docente en Universidad Laica Eloy \\ Alfaro de Manabí (ULEAM). Manta, Ecuador. \\ https://orcid.org/0000-0001-5508-924X \\ carmita.alvarez@uleam.edu.ec \\ Autora: Peñafiel-Tircio Karina \\ Licenciada en Trabajo Social / Perito del Consejo de la Judicatura. Manta, Ecuador. \\ https://orcid.org/0000-0002-2992-8246 \\ karinapenafiel@funcionjudicial.gob.ec
}

\begin{abstract}
RESUMEN
Este estudio se realiza mediante el objetivo que permite identificar en la familia su corresponsabilidad en el proceso de la restitución de derechos en los adolescentes en conflicto con la ley en el Cantón Guayaquil 2019-2020, los adolescentes en conflicto con la ley presentan conductas asociales o comportamientos delictivos incurriendo en el cometimiento de delitos ,debido a ello se establecen medidas cautelares y socioeducativas que pueden ser privativas o no privativas de libertad; existiendo una vulneración de sus derechos y la discriminación con la sociedad .Por otra parte en el momento en que el adolescente se encuentra en un proceso de reinserción debería de tener el apoyo tanto del Estado, la familia y la sociedad con el fin de lograr una reinserción familiar asertiva sin que preceda incluso a la reincidencia de los delitos. La investigación tiene un enfoque de intervención social, aplicando el método de investigación cuali-cuantitativo, siendo de tipo descriptivo y bibliográfico que permitirá conocer teóricamente las variables de estudio, a su vez la población objeto de estudio serán los adolescentes, padres de familia y profesionales inmersos en el campo de estudio, en donde se realiza a través de un seguimiento de los adolescentes que se encuentran en un proceso de reinserción en el Cantón Guayaquil y sus familias, la misma que tiene como fin conocer la corresponsabilidad familiar, la influencia 0 aportación que tienen en el adolescente en conflicto con la ley en la restitución de sus derechos y de su reinserción a la familia y a la sociedad..
\end{abstract}

Palabras claves: Adolescentes infractores, Corresponsabilidad familiar, reinserción social, restitución de derechos, seguridad jurídica.

Información del manuscrito:

Fecha de recepción: 09 de noviembre de 2020.

Fecha de aceptación: 04 de enero de 2021.

Fecha de publicación: 11 de enero de 2021. 


\begin{abstract}
This study is carried out by means of the objective that allows to identify in the family their co-responsibility in the process of restitution of the rights of adolescents in conflict with the law in the Guayaquil Canton 2019-2020, adolescents in conflict with the law present asocial behaviors or Criminal behaviors incurring in the commission of crimes, due to which precautionary and socio-educational measures are established, which may be custodial or non-custodial; There is a violation of their rights and discrimination with society. On the other hand, at the time that the adolescent is in a process of reintegration, they should have the support of both the State, the family and society in order to achieve a Assertive family reintegration without even preceding the recidivism of crimes. The research has a focus of social intervention, applying the qualitative-quantitative research method, being descriptive and bibliographic in nature that will allow to theoretically know the study variables, in turn the population under study will be adolescents, parents and professionals immersed in the field of study, where it is carried out through a follow-up of adolescents who are in a process of reintegration in the Guayaquil Canton and their families, the same which aims to know family co-responsibility, influence or contribution that they have in adolescents in conflict with the law in the restitution of their rights and their reintegration into the family and into society.
\end{abstract}

Keywords: Adolescent offenders, Family co-responsibility, social reintegration, restitution of rights, legal security.

\title{
1. INTRODUCCIÓN
}

La situación de los adolescentes en conflicto con la ley, enfocada a los derechos humanos, ha sido de vital importancia, debido que a través de las políticas públicas promueven y garantizan la protección de los derechos de los adolescentes y la restitución de estos, sin embargo, no se ha podido frenar el incremento de delitos cometidos por adolescentes.

En el análisis de la Corporación Británica de Radiodifusión (2016), expone que en América Latina existen cinco países con mayor criminalidad efectuada por adolescentes, como lo son: El Salvador, Colombia, Venezuela, México y Honduras, donde adjudican que esto se debe especialmente al dominio que ejercen las organizaciones de tráfico de drogas, extorsión y robos.

Es por ello por lo que los gobiernos de turno deben asignar fondos públicos para combatir la delincuencia, y sobre todo establecer programas de recuperación y reinserción social.

Al mismo tiempo se conoce que en el Ecuador, en la Unidad Judicial de Adolescentes Infractores (2018), se realizan "las pericias emitidas por la 
autoridad competente se observa a través de la relación directa con el adolescente y su familia, el conocimiento de su entorno familiar y social" (p. 16). En algunas ocasiones se evidencia como conflictivo, debido a las relaciones débiles de comunicación entre los integrantes de la familia y la carencia de establecimientos de normas de convivencias y de límites.

No obstante el problema detectado en el cantón Guayaquil en los adolescentes en conflicto con la ley, existen diversos factores o fenómenos sociales que resultan ser multicausales, en el cual se correlacionan factores individuales, familiares, sociales, culturales y económicos; en donde los adolescentes infractores realizan una serie de actos incurriendo en el cometimiento y en la reincidencia de delitos siendo los principales: el homicidio, abuso sexual, violación y expendio de sustancias catalogadas a fiscalización; "lo que determina por parte de la sociedad una discriminación, aplicación por orden judicial de medidas cautelares o medidas socio educativas privativas o no de libertad y la vulneración de derechos" (Hein, 2016, p. 8).

Algunas investigaciones relacionadas al tema, como lo indica Arellano \& Atanasio:

La influencia de la relación familiar es importante porque desempeña la función de influencias tempranas y duraderas, donde el ambiente familiar es el primero, porque los primeros educadores son los padres, enseñando a sus hijos a socializar y en éste adquiere que sus hijos cuenten con las enseñanzas para enfrentarse al medio, que ingresarán en el transcurso de su vida influyendo en su interacción social, conductual y psicológico de los niños y los adolescentes. (2019, p. 22)

Este estudio plantea su importancia acerca de las relaciones que se mantienen entre los miembros de las familias y los roles que desempeña cada uno de ellos para conocer la corresponsabilidad que tienen ante los actos que realizan los adolescentes que se encuentran en conflicto con la ley, además, se contó con el apoyo de las Casas de acogida de los adolescentes infractores involucrados en la restitución social, donde se determinó el problema de estudio y la afectación que tiene la corresponsabilidad de la familia. 
Se empleó este estudio mediante la revisión teórica acerca de diversas conceptualizaciones relacionadas a la problemática principal, en donde se encuentra que la corresponsabilidad es una de las principales problemáticas de hijos adolescentes, su impacto en las familias y sus equilibrios en distribución de roles y particularmente en la restitución de derechos de los adolescentes en conflicto con la Ley.

Para establecer el propósito investigativo se planteó el siguiente objetivo que permite identificar en la familia su corresponsabilidad en el proceso de la restitución de derechos en los adolescentes en conflicto con la ley en el cantón Guayaquil 2019-2020. Al mismo tiempo se establecen directrices investigativas que permiten conocer la influencia de las relaciones familiares en el adolescente en la restitución de sus derechos; determinar el nivel de conocimiento del adolescente con respecto a su seguridad jurídica en cuanto al cometimiento de delitos, la discriminación y vulneración de sus Derechos, y por último investigar la influencia que tiene el entorno social del adolescente en el cometimiento de delitos, la discriminación y la vulneración de sus derechos.

Esto se fundamentó directamente en la formulación de la pregunta de investigación ¿Cuál es la corresponsabilidad familiar para el proceso de la restitución de derechos en los adolescentes en conflicto con la ley en el Cantón Guayaquil 2019-2020?

\section{REVISIÓN LITERARIA}

La corresponsabilidad o el reparto de responsabilidades dependientes, con el fin de distribuir justamente los tiempos de vida de mujeres y hombres. Es compartir en igualdad las responsabilidades familiares.

Ávila afirma que "se debe alcanzar los resultados esperados en establecer la corresponsabilidad de padres ante las acciones de los hijos, donde lo obliga a trabajar sobre tres dimensiones básicas de la corresponsabilidad familiar" (2017, p. 1191), en las que cada actor social debe ser y hacerse protagonista, en cuanto a la distribución de tareas para cada uno de los miembros de la familia, establecer una acción coordinada y armónica entre los miembros de la familia, mejorar el apoyo emocional y motivacional de la familia. 
Cuando hablamos de corresponsabilidad, se refiere al necesario reparto y responsabilidades familiares entre miembros de un hogar: pareja, hijos, hijas $u$ otras personas que convivan en el mismo domicilio.

En las acciones que comprende la corresponsabilidad, se evidencia que es importante establecer las tareas por cada miembro familiar, mantener tareas establecidas, tener el apoyo emocional y motivacional en las tareas encomendadas, donde estas actividades contribuyen a mantener una corresponsabilidad efectiva dentro de la familia.

Según Suárez \& Jama afirma que "para considerar el éxito o fracaso al momento de abordar adolescentes infractores de la ley penal, es importante que las familias tengan un acompañamiento, para que puedan contribuir a una guía para el control de los adolescentes" (2017, p. 56).

El abordar a los adolescentes se constituye en herramienta fundamental para las instituciones de reeducación, y su efecto tiene gran impacto cuando las redes familiares se vuelven fuente de apoyo básico y proveen las condiciones adecuadas para que puedan concretar sus metas de vida, obrando con rectitud.

Es necesario comprender que las leyes establecidas, deben ser una guía fundamental que muchos padres deben recalcar a sus hijos desde temprana edad, tanto como no robar o hurtar, tener valores y principios, son pilares fundamentales que debe establecer toda familia.

Sánchez, Aguirre \& Solano, para referirse a las corresponsabilidades propias y claramente diferenciadas de todos los actores sociales, para la garantía de los derechos quienes asumen "la corresponsabilidad familiar como los repertorios comportamentales que median en la división, reparto, participación y aprobación en relación con los quehaceres y encargos entre los miembros de una familia" (2015, p.117).

Es necesario conocer en base a lo expuesto que la corresponsabilidad debe ser integrada por principios y elementos que actúan mejorando el desempeño del sistema a través del apoyo constante, el trato dignificante, el amor, el respeto y la aceptación del otro. 
Tal como fundamentan la corresponsabilidad familiar, se induce a mantener una autoridad, donde establezcan tareas o actividades a cada miembro de su familia, esto con el fin de crear conciencia en cada individuo donde deben comprender que se debe ejercer tareas que suplirán sus necesidades a un futuro.

No obstante, desde la noción de la reinserción social Montejano, Galán, De la Rosa \& Rodríguez afirman que "la familia se constituye en motor de la corresponsabilidad porque es unidad ecosistémica favorecedora de supervivencia, evolución y cambio, es el ámbito originario para la consolidación y crecimiento de la libertad" (2019, p. 223)

Para conocer específicamente sobre la reinserción, es escenario educar con principio y valores a cada adolescente, para el entramado de relaciones interpersonales que protege y promueve el descubrimiento de la propia identidad a través de metodologías pedagógicas que subyacen a la cotidiana convivencia.

Las familias son un eje central para la reinserción de los adolescentes que se encuentran en conflicto con la sociedad, debido a que, en el estado actual de mantenerse privados de su libertad, lo único que buscan es tener algo de afectividad, comprensión, entendimiento por parte de los padres.

Las relaciones familiares según Arias "llevan a que las relaciones dentro de la familia, al contemplar acciones de corresponsabilidad se sometan a un proceso de construcción donde existen fisuras de percepción entre los sujetos adultos de la familia, que atribuye a mejorar el desarrollo familiar" (2015, p. 135).

Al representar una figura de respeto dentro del hogar los padres pueden mantener buenas relaciones familiares, pudiendo establecer una seguridad, ayudar a superar las dificultades, a solucionar los problemas o conflictos, dichos actos influyen en el mejoramiento de las relaciones familiares.

Se ha planteado hasta el momento la importancia de las relaciones familiares, sin embargo, es importante reconocer que esto es un proceso de interacción entre los miembros de la familia, que a partir de estas interacciones se establecen lazos que les permiten a los miembros de la familia permanecer unidos y luchar por alcanzar metas propuestas. 
Los factores de riesgo y protección que se dan en la familia que promueven o desmotivan el comportamiento delictivo del adolescente se manifiestan mediante los problemas en los que se pueden ver envueltos los jóvenes, uno de los que suscita mayor preocupación y temor por parte de la opinión pública, es la delincuencia y la violencia. (Galván \& Durán, 2019, p. 88)

Se han estudiado un sin número de variables de riesgo que anteceden al desarrollo del comportamiento delictivo como factores sociales, familiares, las influencias de los iguales y ciertas características cognitivas. De hecho, durante esta etapa evolutiva parece ser que los jóvenes exploran y experimentan diversos comportamientos de riesgo.

De ahí, que sea importante poder distinguir aquellas conductas de riesgo de carácter experimental, que se manifiestan como parte del desarrollo y que cesan con el tiempo, de aquellas que persisten y se pueden volver más crónicas.

"La delincuencia puede señalarse como un conjunto de variables asociadas a un hecho social, que responden a clasificaciones, tipologías y elaboraciones provenientes de determinadas concepciones del ser humano" (Suárez \& Jama, 2015).

En tanto a otras variables intervinientes asociadas al problema de la delincuencia pueden ser desconocidas aún.

Se comprende que la desorganización, desintegración social y disolución de las redes de apoyo social y de grupos sociales primarios hechos presentes en las sociedades industriales y urbanas modernas, sería un factor clave en la génesis de problemas psicosociales como la delincuencia.

La interacción pobre entre padres-hijos y los conflictos maritales son considerados como factores de riesgo familiares para la conducta antisocial y delictiva. Se ha planteado que si en una familia la relación es cálida y afectuosa la probabilidad de aparición de la conducta problemática disminuye. (Sanabria \& Uribe, 2015, p. 257)

Cabe mencionar que en un medio familiar propicio proporciona las herramientas para una adecuada relación intrafamiliar, sin embargo, un interior familiar 
conflictivo puede generar límites en el aprendizaje de estrategias para la resolución de problemas.

Además, la interacción puede manifestarse una inadecuada estabilidad emocional, en los niños que se encuentran en su etapa de formación y en los adolescentes por su actitud desafiante de la autoridad de los padres.

"La adolescencia es una etapa de transición que no tiene límites temporales fijos" (Belda, 2015, p. 57). Ahora bien, los cambios que ocurren en este momento son tan significativos que resulta útil hablar de la adolescencia como un período diferenciado del ciclo vital humano. Este período abarca desde cambios biológicos hasta cambios de conducta y status social, dificultando de esta manera precisar sus límites de manera exacta.

Consecuentemente se puede precisar porqué la reincidencia está marcada por unas problemáticas de índole individual, familiar y social, las cuales desarrollaremos a continuación:

Dentro de las causas individuales que originan la proliferación de la reincidencia se encuentra el deseo de mantener el estilo de vida que el adolescente creó a partir de la obtención de dinero fácil con el pleno conocimiento de que el hurto, el homicidio, y el tráfico y porte de estupefacientes, son delitos menores que tienen sanciones reducidas, beneficios e incluso la posibilidad de quedar en libertad. (Montejano, Galán, \& De la Rosa, 2019, p. 223)

Las causas que acompaña la reincidencia es el consumo de sustancias psicoactivas; especialmente en los delitos cometidos bajo efectos de una droga puede aumentar o estimular la excitabilidad, la irritabilidad o las disposiciones violentas por parte de quien comete el acto delictivo.

Los profesionales consideran que el consumo de sustancias psicoactivas es un factor determinante que moviliza a los adolescentes a reincidir en conductas delictivas.

Como se deduce del enunciado hay una relación entre consumo y actos delictivos, al respecto hay que precisar con relación a este tema que existen dos tipos de delincuencia, una funcional y otra inducida. 
La explotación laboral, es otro factor desencadenante en la reincidencia en los adolescentes, en el entendido que el "trabajo infantil", es toda actividad que priva a los adolescentes, de una vida tranquila, de su dignidad, que es nocivo para su desarrollo físico y psicológico. (Reyes, 2018, p. 66)

Las dificultades económicas, entre las que se destacan, el desempleo de los integrantes de la familia del adolescente, las condiciones de precariedad en las cuales están inmersos, son detonantes a la reincidencia.

No obstante, es importante conocer la seguridad jurídica en cuanto al cometimiento de delitos, la discriminación y vulneración de sus derechos.

El Sistema de responsabilidad penal para adolescentes surge de una problemática social como un modelo de administración de justicia que reconoce al adolescente como sujeto de derechos y resalta cambios fundamentales en el principio de la protección integral; este sistema se interesa en la conducta punible y sus consecuencias antes que en el delito y la pena; persigue que el adolescente tome conciencia de su conducta e implicaciones para su proyecto de vida, el de la víctima, la comunidad y la sociedad en general. (Constitución de la República del Ecuador, 2008)

La realidad ecuatoriana en materia de justicia restaurativa acude al desarrollo de ciertos conceptos de profunda discusión y debate por parte de académicos, líderes y todos aquellos interesados en hacer de la justicia una propuesta más acertada para la población de niños y adolescentes.

No obstante, afirma Ávila (2017) que para poder entender el porqué de algunas conductas y actitudes que tienen los adolescentes, es pertinente definir que es la adolescencia:

Estipulado en las reglas mínimas de las Naciones Unidas para la administración de la justicia de menores según las Reglas de Beijing (1985), hace mención en el inciso 3. Ampliación del ámbito de aplicación de las Reglas, refiriéndose en los incisos 3.1, 3.2 y 3.3., se conoce que los arreglos importantes de las Reglas no solo se aplicarán a los adolescentes culpables, sino también a los menores que podrían ser procesados por completar una demostración particular que no es 
culpable debido a una conducta adulta, donde se realizarán esfuerzos para ampliar la extensión de los estándares contenidos en las Reglas a todos los menores recordados por las estrategias identificadas con la atención del niño y la asistencia del gobierno.

Consecuentemente se reconoce que el Estado Ecuatoriano, mediante la intervención de varias instituciones públicas y privadas, realiza esfuerzos para ampliar el alcance de los estándares contenidos en las leyes que deben conocer los adolescentes.

El Código de la Niñez y la Adolescencia incorpora una clasificación de los derechos de los niños, niñas y adolescentes, la cual apremia que los derechos incluyen la protección contra todo tipo de malos tratos, abandono, explotación y crueldad, e incluso el derecho a una protección especial en tiempos de guerra y protección contra los abusos del sistema penal. (Código de la Niñez y la Adolescencia, 2008: Art. 16).

La Comisión Interamericana de Derechos Humanos reconoce que la Convención sobre los Derechos del Niño, junto con estos y otros instrumentos internacionales, constituyen un corpus iuris internacional para la protección de los niños niñas y adolescentes, que puede servir como "guía interpretativa" a la hora de hacer efectivos sus derechos.

En cuanto al entorno social Reyes afirma en su artículo sobre "Integración social en los adolescentes" que "el entorno social influye en el desarrollo cognitivo y emocional del adolescente, en su adaptación al entorno, en el aprendizaje de actitudes y valores, en la formación de la identidad, en la adquisición de habilidades sociales" (2018, p. 66)

Esta influencia ocurre porque los amigos, además de formar parte de la pandilla, contribuyen, como cohorte, a transmitir las actitudes o los comportamientos normativos de una generación particular.

Por lo tanto, la familia es considerada como la unidad básica en la formación del niño como del adolescente siendo el constructor de su personalidad por medio de comunicación, afectos, compartir actividades, asumir responsabilidades, promoviendo el relacionamiento directo con la sociedad. 


\section{MATERIALES Y MÉTODOS}

\section{a) Diseño metodológico.}

El proceso de investigación -acción realizado, tuvo un enfoque de investigación mixta, debido a que se aplicó a los adolescentes en conflicto con la ley y a sus familias.

El tipo de investigación fue descriptiva, debido a que se trabajó sobre realidades de hechos, y su característica fundamental fue la de presentar una interpretación correcta. Para la investigación descriptiva, es primordial descubrir algunas características fundamentales de conjuntos homogéneos de fenómenos según la aplicación de las variables de estudios teniendo relación estrecha con los objetivos planteados en la investigación.

En lo que respecta al método se hizo de manera deductiva, analizando desde lo macro a lo micro.

\section{b) Participantes de la Investigación}

La población fue de 706 adolescentes que se encuentran en conflicto con la ley en el Cantón Guayaquil en los Centros de atención integral Masculino, Centro de atención integral Femenino, Casa de acogida "Libertadores" y Casa de acogida "Juan Elías", quienes fueron la población; se aplicó la formula muestral probabilística la que dio como resultado 249 adolescentes que fueron encuestados, no obstante, se tomó a consideración 706 familias que arrojó el mismo número 249 de familias que fueron encuestadas.

\section{c) Área de estudio.}

Este estudio fue aplicado en los Centros de Rehabilitación para adolescentes del cantón de Guayaquil: Centros de atención integral Masculino, Centro de atención integral Femenino, Casa de acogida "Libertadores" y Casa de acogida "Juan Elías". 


\section{d) Técnicas empleadas.}

Se utilizó como herramientas la encuesta mediante un cuestionario de preguntas cerradas aplicadas a los adolescentes y padres de familia, y las entrevistas aplicadas a profesionales en el campo de Trabajo Social.

Los instrumentos se aplicaron de manera presencial y semi presencial, utilizando como base el uso de formularios de Google/ Gmail con el siguiente enlace a los padres de

familia: https://docs.google.com/forms/d/e/1FAlpQLScSCtSgq7nQACrVBIhaoOHcmMF Q0KKU_HX6B7qrG0mL1YihSg/viewform

\section{e) Análisis estadísticos.}

Estos datos fueron ingresados al programa SPSS, el mismo que permitió realizar un análisis correlacional de las variables en un estudio representado mediante tablas que dan como resultado el reconocimiento de la influencia de las relaciones familiares en el proceso de restitución de derechos en los adolescentes en conflicto con la ley, de igual modo se pudo establecer el conocimiento sobre los derechos que amparan a los adolescentes y la influencia que tiene el entorno social.

\section{RESULTADOS}

Dentro de los resultados, obtenidos para conocer la influencia de las relaciones familiares en el adolescente en la restitución de sus derechos, datos analizados mediante la encuesta a padres de familia, adolescentes mediante un cruce de variables demográficos y los resultados de las entrevistas aplicadas a profesionales que se detalla en la tabla 1 y 2 .

Tabla 1. Influencia de las relaciones familiares en el proceso de restitución de derechos en los adolescentes en conflicto con la ley.

\begin{tabular}{|c|c|c|c|c|c|}
\hline & 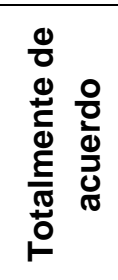 & 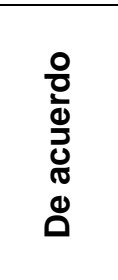 & 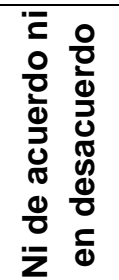 & 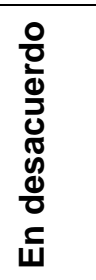 & 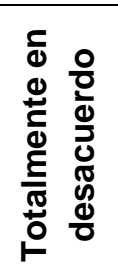 \\
\hline 1 a 3 persona & $100 \%$ & ,0\% &, $0 \%$ &, $0 \%$ & $0 \%$ \\
\hline 3 a 5 personas & $23,3 \%$ & $60,5 \%$ & $16,3 \%$ & ,0\% & ,0\% \\
\hline
\end{tabular}




\begin{tabular}{rrrrrr} 
Más de 5 personas &, $0 \%$ &, $0 \%$ & $16,4 \%$ & $47,1 \%$ & $36,5 \%$ \\
\hline TOTAL & $10,8 \%$ & $10,4 \%$ & $15,3 \%$ & $35,7 \%$ & $27,7 \%$ \\
\hline
\end{tabular}

Fuente: Autoras (2021).

Se puede constatar que un $100 \%$ de padres de familia afirman donde viven en su hogar de 1 a 3 personas están totalmente de acuerdo en que las relaciones familiares influyen en el proceso de restitución de derechos de los adolescentes en conflicto con la ley, mientras que un $60.5 \%$ en familias que están conformadas por 3 a 5 personas están de acuerdo, y un $47.1 \%$ en familias conformadas en más de 5 personas están en desacuerdo y un $36.6 \%$ están totalmente en desacuerdo.

Tabla 2. Influencia de las relaciones familiares contribuyen en la restitución de sus derechos.

\begin{tabular}{|c|c|c|c|c|c|}
\hline & 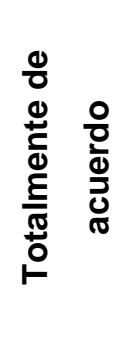 & 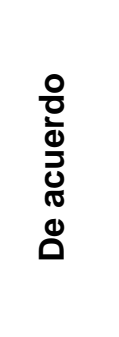 & 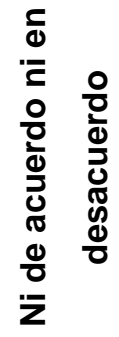 & 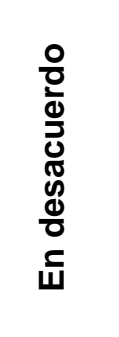 & 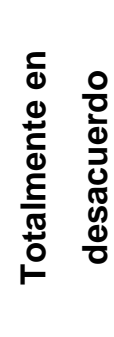 \\
\hline 14 a 15 años & $100 \%$ &, $0 \%$ &, $0 \%$ &, $0 \%$ &, $0 \%$ \\
\hline 16 a 17 años & $51,8 \%$ & $48,2 \%$ &, $0 \%$ &, $0 \%$ &, $0 \%$ \\
\hline 17 a 18 años &, $0 \%$ & $37,6 \%$ & $24,1 \%$ & $27,7 \%$ & $10,6 \%$ \\
\hline TOTAL & $27,3 \%$ & $37,3 \%$ & $13,7 \%$ & $15,7 \%$ & $6,0 \%$ \\
\hline
\end{tabular}

Fuente: Autoras (2021).

Los adolescentes en edades de 14 a 15 años en un 100\% afirman estar totalmente de acuerdo en que la influencia de las relaciones familiares contribuye en la restitución de sus derechos, mientras que un $51.8 \%$ de adolescentes en edades de 16 a 17 años mencionan estar totalmente de acuerdo, de igual manera un $48.2 \%$ están de acuerdo con respecto a la influencia de las relaciones familiares contribuye en la restitución de sus derechos.

En cuanto a la entrevista a profesionales afirman que, si es importante la influencia de las relaciones familiares del adolescente en la restitución de sus derechos, donde la familia establece un acogimiento, cumpliendo con un rol fundamental en su formación por ende de la restitución de los derechos que han 
sido vulnerados, de igual modo es necesario conocer que las responsabilidades de los padres y miembros de la familia.

Por lo consiguiente se presenta la comprobación del objetivo determinar el nivel de conocimiento del adolescente con respecto a su seguridad jurídica en cuanto al cometimiento de delitos, la discriminación y vulneración de sus derechos, donde se presentan los siguientes resultados en la tabla 3 y 4 y los resultados de las entrevistas a profesionales.

Tabla 3. Conocimiento sobre los derechos que amparan a los adolescentes.

\begin{tabular}{|c|c|c|c|c|c|}
\hline & 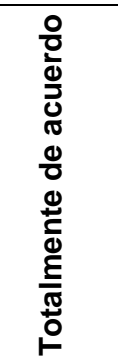 & 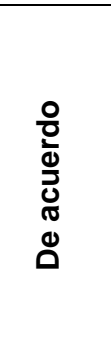 & 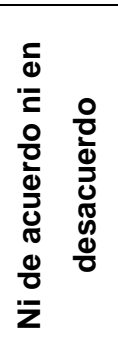 & 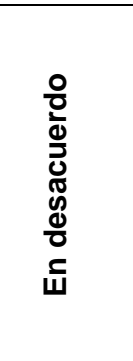 & 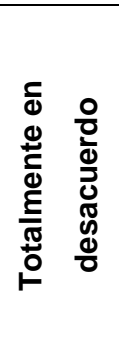 \\
\hline 1 a 3 personas & $58,8 \%$ & $35,3 \%$ & $5,9 \%$ & ,0\% &, $0 \%$ \\
\hline 3 a 5 personas &, $0 \%$ & ,0\% & $18,6 \%$ & $81,4 \%$ &, $0 \%$ \\
\hline Más de 5 personas &, $0 \%$ &, $0 \%$ &, $0 \%$ & $65,6 \%$ & $34,4 \%$ \\
\hline TOTAL & $4,0 \%$ & $2,4 \%$ & $3,6 \%$ & $63,9 \%$ & $26,1 \%$ \\
\hline
\end{tabular}

Fuente: Autoras (2021).

Se evidencia que el $65.6 \%$ de las familias encuestadas conformadas con 3 a 5 personas afirman estar en desacuerdo que su hijo/a posee el conocimiento necesario sobre los derechos que amparan a los adolescentes en conflicto con la ley, mientras que el 65.6\% conformadas por más de 5 personas afirman estar en desacuerdo, mientras un $58.8 \%$ de familias conformadas por 1 a 3 personas aseguran estar totalmente de acuerdo y un 35.3\% están de acuerdo.

Tabla 4. Seguridad jurídica en cuanto al cometimiento de delitos, la discriminación y vulneración de sus Derechos.

\begin{tabular}{|c|c|c|c|}
\hline & $\begin{array}{l}\frac{0}{0} \\
\frac{0}{0} \\
\text { De }\end{array}$ & \& & $\begin{array}{l}\frac{\pi}{0} \\
\frac{\pi}{2}\end{array}$ \\
\hline 14 a 15 años & $28,0 \%$ & $68,0 \%$ & $4,0 \%$ \\
\hline 16 a 17 años &, $0 \%$ &, $0 \%$ & $100 \%$ \\
\hline 17 a 18 años &, $0 \%$ &, $0 \%$ & $100 \%$ \\
\hline TOTAL & $2,8 \%$ & $6,8 \%$ & $90,4 \%$ \\
\hline
\end{tabular}

Fuente: Autoras (2021). 
El 100\% de adolescentes en edades de 16 a 18 años afirman no conocer nada cuál es su seguridad jurídica en cuanto al cometimiento de delitos, la discriminación y vulneración de sus derechos, mientras que un $68 \%$ en edades de 14 a 15 años afirman conocer poco y un $28 \%$ mencionan conocer mucho.

De igual manera los profesionales encuestados hacen referencia que los adolescentes no tienen conocimiento de la seguridad jurídica en cuanto al cometimiento de delitos, la discriminación y vulneración de sus derechos, previo a ello se evidencia un total desconocimiento sobre la seguridad jurídica.

En concordancia se logra comprobar el objetivo que es investigar la influencia que tiene el entorno social del adolescente en el cometimiento de delitos, la discriminación y la vulneración de sus derechos, mismo que se comprueba en la encuesta a las familias, adolescentes y profesionales.

Tabla 5. Influencia del entorno social del adolescente puede incidir en el cometimiento de delitos.

\begin{tabular}{|c|c|c|c|c|c|}
\hline & 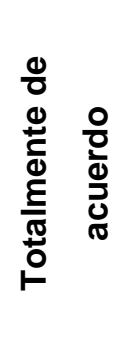 & 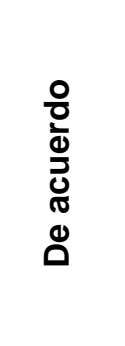 & 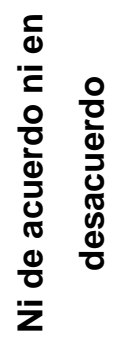 & 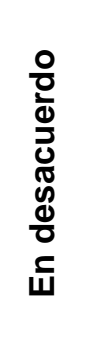 & 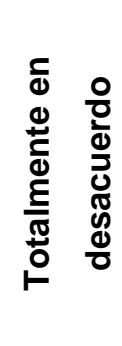 \\
\hline 1 a 3 personas & $100 \%$ & ,0\% & ,0\% & ,0\% & ,0\% \\
\hline 3 a 5 personas & $100 \%$ &, $0 \%$ &, $0 \%$ &, $0 \%$ &, $0 \%$ \\
\hline Más de 5 personas & $33,3 \%$ & $47,1 \%$ & $7,9 \%$ & $6,3 \%$ & $5,3 \%$ \\
\hline TOTAL & $49,4 \%$ & $35,7 \%$ & $6,0 \%$ & $4,8 \%$ & $4,0 \%$ \\
\hline
\end{tabular}

Fuente: Autoras (2021).

Se puede constatar que el $100 \%$ de las familias encuestadas conformadas por 1 a 5 personas están totalmente de acuerdo en que el entorno social del adolescente puede incidir en el cometimiento de delitos, la discriminación y la vulneración de sus derechos, mientras que el $47.1 \%$ de familias conformadas por más de 5 personas afirman estar de acuerdo y un 33.3\% afirman estar totalmente de acuerdo. 
Tabla 6. El entorno social influye en el cometimiento de delitos.

\begin{tabular}{|c|c|c|c|c|c|}
\hline & 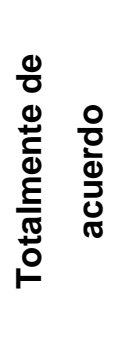 & 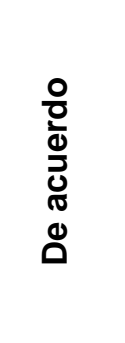 & 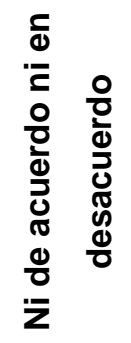 & 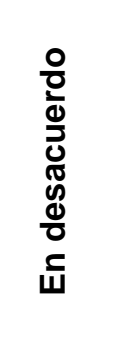 & 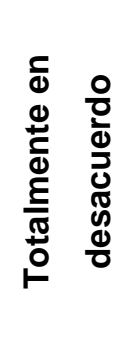 \\
\hline 14 a 15 años & $92,0 \%$ & $8,0 \%$ & ,0\% & ,0\% &, $0 \%$ \\
\hline 16 a 17 años &, $0 \%$ & $49,4 \%$ & $50,6 \%$ & ,0\% &, $0 \%$ \\
\hline 17 a 18 años &, $0 \%$ &, $0 \%$ & $9,9 \%$ & $22,0 \%$ & $68,1 \%$ \\
\hline TOTAL & $9,2 \%$ & $17,3 \%$ & $22,5 \%$ & $12,4 \%$ & $38,6 \%$ \\
\hline
\end{tabular}

Fuente: Autoras (2021).

Se puede evidenciar que un $92 \%$ de los adolescentes encuestados en edades de 14 a 15 años afirman estar totalmente de acuerdo en que el entorno social influye en el cometimiento de delitos, la discriminación y la vulneración de los derechos, mientras que el $68.1 \%$ en edades de 17 a 18 años mencionan estar totalmente en desacuerdo, en cuanto al 50.6\% de adolescentes en edades de 16 a 17 años afirman estar ni de acuerdo ni en desacuerdo y un $49.4 \%$ afirman estar de acuerdo.

En consecuencia, los profesionales responden que el entorno social del adolescente si influye en el cometimiento de delitos, discriminación y la vulneración de sus derechos, esto debido a entrevistas realizadas donde pudieron evidenciar que la mayoría crecen en hogares disfuncionales, donde no se ha establecido roles ni reglas, considerando también que desde niños van viendo los actos que los adultos realizan, ya sean por medios de comunicación o por su entorno.

\section{DISCUSIÓN}

En base a los resultados obtenidos se pudo conocer que los padres de familia encuestados consideran estar totalmente de acuerdo en que las relaciones familiares influyen en el proceso de restitución de derechos de los adolescentes en conflicto con la ley, no obstante se evidenció que del mismo modo están de acuerdo en que las relaciones familiares contribuyen en la restitución de sus 
derechos, donde la familia cumplen con un rol fundamental en la inducción del estado en el cual se encuentran los adolescentes, manteniendo las responsabilidades por parte de los padres y miembros de la familia.

Tal como muestra Arias (2015), que las relaciones familiares complementan acciones de corresponsabilidad, que se someten a un proceso de construcción donde existen fisuras de percepción entre los sujetos adultos de la familia, pudiendo establecer una seguridad, ayudar a superar las dificultades, a solucionar los problemas o conflictos, actos que influyen en el mejoramiento de las relaciones familiares.

Es importante mediar en las acciones de reinserción de los adolescentes, para ello el Estado Ecuatoriano debe regular varias leyes para que los adolescentes tengan el pleno reconocimiento de sus derechos, de ahí todas las medidas, acciones, programas y legislación que así lo hagan deberá efectuarse con la ayuda de la familia que debe realizar el acompañamiento necesario para reforzar las relaciones familiares, pero sobre todo mejorar la afectividad y seguridad como familia.

Se pudo determinar que las familias encuestadas conformadas con 3 a 5 personas afirman estar en desacuerdo que los hijos poseen el conocimiento necesario sobre la seguridad jurídica en cuanto al cometimiento de delitos, la discriminación y vulneración de sus derechos, esto es afirmado de igual modo en la encuesta a los adolescentes donde responden que no conocen nada, consecuentemente los profesionales reafirman que los adolescentes no tienen conocimiento de la seguridad jurídica, previo a ello dentro de los centros de rehabilitación, realizan talleres educativos referentes a la seguridad jurídica, optando por realizar talleres referentes en los diferentes contextos; pero para un mejor entendimiento sobre los Derechos y la Obligaciones del ciudadano.

A ello se toma como referente la seguridad jurídica en cuanto al cometimiento de delitos, la discriminación y vulneración de los derechos de los adolescentes, como lo estipula la Constitución de la República del Ecuador (2008) que el sistema de responsabilidad penal para adolescentes surge de una problemática social como un modelo de administración de justicia que reconoce al adolescente 
como sujeto de derechos y resalta cambios fundamentales en el principio de la protección integral.

Es importante que los padres de familia sean guías de sus propios hijos en cuanto a su formación en valores, debido a que deben comprender desde temprana edad, que el sistema legal ecuatoriano, se interesa en la conducta punible y sus consecuencias antes que, en el delito y la pena, a ello, se deberá plasmar una conciencia en la conducta e implicaciones del proyecto de vida de los adolescentes, comunidad y sociedad en general.

Se pudo conocer que las familias están totalmente de acuerdo en que el entorno social del adolescente puede incidir en el cometimiento de delitos, la discriminación y la vulneración de sus derechos, al igual que los adolescentes afirman estar totalmente de acuerdo en que el entorno social influye en el cometimiento de delitos, en consecuencia, los profesionales responden que el entorno social del adolescente si influye en el cometimiento de delitos, esto se debe a su familia se conforma por familias disfuncionales, donde no se ha establecido roles ni reglas.

Reyes (2018) afirma que el entorno social influye en el desarrollo cognitivo y emocional del adolescente, en su adaptación al entorno, en el aprendizaje de actitudes y valores, en la formación de la identidad, en la adquisición de habilidades sociales.

Por lo tanto, la familia es considerada como la unidad básica en la formación del adolescente siendo el constructor de su personalidad por medio de comunicación, afectos, compartir actividades, asumir responsabilidades, promoviendo el relacionamiento directo con su entorno social reafirmando que el entorno social del adolescente influye en el cometimiento de delitos, la discriminación y la vulneración de sus derechos.

\section{CONCLUSIONES}

1. Se pudo concluir que los padres de familia como adolescentes son conscientes que las relaciones familiares influyen en el proceso de restitución de derechos de los adolescentes en conflicto con la ley, donde se conoce que la familia juega un rol fundamental en la inducción del estado en el cual se 
encuentran los adolescentes, previo a ello se pudo conocer que varias familias no realizan el proceso de intervención y acompañamiento de las actividades que se realizan en los Centros de atención integral masculinos y femeninos.

2. Los adolescentes carecen del conocimiento sobre la seguridad jurídica en cuanto al cometimiento de delitos, la discriminación y vulneración de sus derechos, pese a que se realizan talleres referentes a la seguridad jurídica, varios adolescentes no le dan la debida importancia en conocer cuáles son sus derechos amparados por la ley.

3. Se constató que el entorno social del adolescente incide en el cometimiento de delitos, la discriminación y la vulneración de sus derechos, donde varios adolescentes afirman que debido a su entorno pudieron inducirse al cometimiento de delitos, inclusive detectando un factor que es pertenecer a familias disfuncionales donde no se ha establecido roles ni reglas.

\section{LIMITACIONES Y RECOMENDACIONES}

Dentro de las limitaciones que se presentaron, estuvo la gestión documental para proceder al levantamiento de información en los adolescentes, donde directores de los centros, no permitieron efectuar el estudio hasta no tener la aprobación y cobertura por parte de los directivos de los Centros de atención integral Masculino, Centro de atención integral Femenino, Casa de acogida "Libertadores" y Casa de acogida "Juan Elías", pese a ello fue necesario tomar las debidas medidas de protección con la salud debido al confinamiento por el COVID 19.

1. Se recomienda que se deba crear charlas dirigidas a los adolescentes que se encuentran en los Centros de atención integral masculino y femenino, sobre el proceso de restitución de derechos de los adolescentes en conflicto con la ley.

2. Es importante crear actividades que generen una conciencia social, junto con el apoyo y acompañamiento de las familias de adolescentes donde se trate de generar un contraste de vivencias que pudieran afectar a sus acciones, de tal modo que se formen individuos consientes sobre la seguridad jurídica en cuanto al cometimiento de delitos, la discriminación y vulneración de sus derechos. 


\section{REFERENCIAS}

Acunso-Garantías del adolescente infractor, T708 (Unidad Judicial de Adolescentes Infractores 13 de Noviembre de 2018).

Adolescencia, C. d. (2008). Art. 16. Quito, Ecuador: Registro Civil.

Alcántar, V. M., Maldonado, S., \& Arcos, J. (2017, p. 56). Medición de clima laboral requerido para asegurar la efectividad del sistema de gestión de calidad. Revista Internacional de Administración y Finanzas, 5(3), 55-68.

Arellano, D. C., \& Atanacio, R. M. (2019, p. 22). Relaciones intrafamiliares de estudiantes del nivel secundario de una institucion pública y privada. Huacho - Perú: Universidad Nacional José Faustino Sánchez Carrión.

Arias, J. V. (2015, p. 135). La resocialización y la reincidencia de adolescentes en conductas delictivas en el Departamento de Caldas Colombia. Revista suma luris, 135 - 139.

Ávila, N. V. (2017, p. 1191). Corresponsabilidad familiar en instituciones de reeducación para adolescentes infractores. Revista Latinoamericana de Ciencias Sociales, Niñez y Juventud, pp. 1191-1206.

Ávila, R. J. (2017). Experiencias de vida en el proceso de reinserción social de jóvenes que han finalizado su rehabilitación en un centro de recuperación privado de la ciudad de Guayaquil. Guayaquil, Ecuador: Universidad Catolica de Santiago de Guayaquil.

Belda, U. R. (2015, p. 57). Técnicos Especialistas de Menores de la Generalitat Valenciana. Valencia, España: MAD.

Constitucion de la República del Ecuador. (2008). Registro Oficial N.449. Quito, Ecuador.

Corporación Británica de Radiodifusión. (2016). Cuáles son los 6 países de América Latina que están entre los 13 con peores índices de criminalidad en el mundo. Obtenido de https://www.bbc.com/mundo/noticias-americalatina-38171437

Galván, M. A., \& Durán, P. N. (2019, p. 88). Adolescentes infractores y promoción de acciones prosociales: una tarea pendiente. Revista de Ciencias sociales.Editorial El Ágora, 88-93.

Hein, A. (2016, p. 8). Factores de riesgo y delincuencia juvenil, revisión de la literatura nacional e internacional. México: Fundación Paz Ciudadana.

Montejano, T. L., Galán, J. J., \& De la Rosa, R. P. (2019, p. 223). Reinserción social de adolescentes en conflicto con la ley. Un estudio conceptual. Estudios Socio-jurídicos, 22(1), 233-234. Un estudio conceptual. Estudios Socio-jurídicos, 22(1), 233-234. 
Reglas de Beijing. (1985). Reglas mínimas de las Naciones Unidas para la Administración de Justicia de Menores. Asamblea General en su Resolución 40/33 de 1985.

Reyes, S. R. (2018, p. 66). Integración social en los adolescentes. Dialnet, 66124.

Sanabria, A. M., \& Uribe, R. A. (2015, p. 257). Factores psicosociales de riesgo asociados a conductas problemáticas en jóvenes infractores y no. Diversitas: Perspectivas en Psicología, vol. 6, núm. 2,, pp. 257-274.

Sánchez, G., Aguirre, M., \& Solano, N. (2015, p.117). Sobre la dinámica familiar. Revisión documental. Cultura Educación y Sociedad6(2), 117-138.

Suárez, V. H., \& Jama, Z. V. (2015). Contexto sociofamiliar de adolescentes ecuatorianos perpetradores de actividades delictivas. MEDISAN vol.19 no.6 Santiago de Cuba j, 861-844. 